\title{
‘Candidatus Phytoplasma mali’ Genome Encodes a Protein that Functions as an E3 Ubiquitin Ligase and Could Inhibit Plant Basal Defense
}

\author{
Alisa Strohmayer, ${ }^{1}$ Mirko Moser, ${ }^{2}$ Azeddine Si-Ammour, ${ }^{2}$ Gabi Krczal, ${ }^{1}$ and Kajohn Boonrod ${ }^{1,+}$ \\ ${ }^{1}$ RLP AgroScience GmbH, AIPlanta-Institute for Plant Research, Breitenweg 71, 67435 Neustadt an der Weinstraße, Germany \\ ${ }^{2}$ Genomics and Biology of Fruit Crop Department, Research and Innovation Centre, Fondazione Edmund March, San Michele \\ All'Adige, Italy
}

Accepted 24 June 2019.

\begin{abstract}
Phytoplasmas are the causative agent of numerous diseases of plant species all over the world, including important food crops. The mode by which phytoplasmas multiply and behave in their host is poorly understood and often based on genomic data. We used yeast two-hybrid screening to find new protein-protein interactions between the causal agent of apple proliferation 'Candidatus Phytoplasma mali' and its host plant. Here, we report that the ' $\mathrm{Ca}$. P. mali' strain PM19 genome encodes a protein PM19_00185 that interacts with at least six different ubiquitin-conjugating enzymes (UBC; E2) of Arabidopsis thaliana. An in vitro ubiquitination assay showed that PM19_00185 is enzymatically active as E3 ligase with A. thaliana E2 UBC09 and Malus domestica E2 UBC10. We show that a nonhost bacteria (Pseudomonas syringae pv. tabaci) can grow in transgenic $A$. thaliana plant lines expressing PM19_00185. A connection of phytoplasma effector proteins with the proteasome proteolytic pathway has been reported before. However, this is, to our knowledge, the first time that a phytoplasma effector protein with $\mathrm{E} 3$ ligase activity has been reported.
\end{abstract}

Keywords: 'Candidatus Phytoplasma mali', E3 ligase, hostpathogen interaction, phytoplasma, plant immunity, ubiquitination, yeast two-hybrid

Phytoplasmas are plant-pathogenic bacteria that are transmitted by insect vectors and reside in the phloem of their plant host. Although the association between phytoplasmas and their insect vector can be mutually beneficial (Sugio et al. 2011b), plants infected with phytoplasmas show a variety of symptoms such as virescence, sterility of flowers, abnormal internode elongation, witches'-broom, and generalized stunting (Bertaccini 2007). Phytoplasmas are the cause of numerous diseases in plants, including important food crops, leading to heavy damage

${ }^{\dagger}$ Corresponding author: K. Boonrod; kajohn.boonrod@agroscience.rlp.de

Funding: We gratefully acknowledge funding from Deutsche Forschungsgemeinschaft, Geschäftszeichen BO 2939/4-1.

*The $\boldsymbol{e}$-Xtra logo stands for "electronic extra" and indicates that one supplementary table is published online.

The author(s) declare no conflict of interest.

๑) 2019 The American Phytopathological Society in agriculture worldwide. 'Candidatus Phytoplasma mali' is the causative agent of apple proliferation (AP) that leads to considerable yield losses and economic damage in apple production all over Europe.

In contrast to mycoplasmas and spiroplasmas, phytoplasmas have resisted all attempts of cultivation in cell-free media. Thus, phytoplasmas are poorly characterized on a physiological and biochemical basis. The complete determination of phytoplasma genomes and the availability of an increasing amount of phytoplasma draft-genome sequences in GenBank (NCBI) give valuable insights into the genetic makeup of these pathogens and provide important background information in phytoplasma research.

Gene prediction algorithms have become more sensitive but it is still difficult to predict gene and protein function accurately from genomic data. A proteomic approach can be helpful to evaluate genomic data. To better understand how phytoplasmas spread throughout the plant after infection and induce specific symptoms, we searched for protein-protein interactions between ' $\mathrm{Ca}$. P. mali' and plant proteins using library-scale yeasttwo hybrid (Y2H) screens. This approach had been successfully applied to identify protein interaction of AY-WB and AP phytoplasma effector protein SAP11 with CIN-related TCP transcription factors (Janik et al. 2017; Sugio et al. 2011a).

Interesting candidate proteins that are used for bait in such studies are typically proteins potentially located on the surface of the phytoplasma or secreted into the host cell and, thus, can come into direct contact with host proteins. In the case of SAP11, potential effector proteins were found by searching the AY-WB genome for proteins with N-terminal signal peptide (SP) sequences (Bai et al. 2009). Proteins with N-terminal SP are secreted by the Sec-dependent pathway and can directly interact with the host cell. However, in our study, we searched for proteins that contain a transmembrane region and are potentially located outside of the phytoplasma cell. We selected 23 candidate proteins of ' $\mathrm{Ca}$. P. mali' that were undescribed thus far and annotated as hypothetical proteins, with at least one potential transmembrane region predicted by Phobius prediction using a combined transmembrane topology and SP algorithm (Käll et al. 2004). We isolated genes corresponding to these proteins from ' $\mathrm{Ca}$. P. mali' strain PM19 that was previously transmitted from field-collected Cacopsylla picta to healthy test plants of Malus $\times$ domestica (Jarausch et al. 2011) and searched for new protein interactions using $\mathrm{Y} 2 \mathrm{H}$ screening with an Arabidopsis thaliana library.

One of the candidate proteins, named PM19_00185 (GenBank: MK552110) according to its corresponding protein 
ATP_RS00185 of the sequenced strain AT (Kube et al. 2008), showed promising results in the $\mathrm{Y} 2 \mathrm{H}$ screen. Therefore, we analyzed this protein further in detail regarding biochemical properties and its effect on basal plant defense mechanisms.

\section{RESULTS}

\section{Prediction of PM19_00185 protein} of 'Ca. P mali' strain PM19.

The gene of the candidate protein studied in this work was derived from 'Ca. P. mali' strain PM19 (Jarausch et al. 2011). The gene product of PM19_00185 is a 23-kDa protein composed of 200 amino acids and has a $92 \%$ identity at the amino acid level compared with the gene product of ATP_RSO0185 of ' $C a$. P. mali' strain AT (accession number WP_041186049) (Kube et al. 2008), which is annotated as a hypothetical protein. The alignment result indicates that a 12-amino-acid stretch is missing and 3 amino acids were substituted in strain PM19 (Fig. 1A). The result of combined transmembrane topology and SP prediction using Phobius predictor (Käll et al. 2004) indicates that PM19_00185 contains a transmembrane region from amino acids 20 to 41, with a cytoplasmic N-terminal area (amino acids 1 to 19) and a noncytoplasmic C-terminal area (amino acids 42 to 200), suggesting that the major part of the protein could be located outside of the phytoplasma cell. However, using SecretomeP 2.0 prediction (Bendtsen et al. 2004), a sequence-based method to predict nonclassical and not SP-triggered secretion, the result yielded a SecP score of 0.95 , with a recommended threshold of 0.5 for bacterial sequences, which suggests strongly that this protein could be secreted into the host plant. This could, for example, be archived by cleavage or digestion of the $\mathrm{N}$-terminal transmembrane region. However, the exact mechanism remains unclear.

For localization studies, a codon-optimized version of the gene was synthesized (GeneCust, Ellange, Luxembourg) to improve expression in plants. To avoid nonsoluble protein expression, amino acids 1 to 41, including the transmembrane region, were deleted. The resulting construct PM19_00185opt$\triangle T M$ was fused to gene green fluorescent protein $(G F P)$ at its $\mathrm{C}$ terminus and transiently expressed under control of a $35 \mathrm{~S}$ promoter in Nicotiana benthamiana using an Agrobacteriummediated expression system. After 2 days, protoplasts were prepared from infiltrated leaves and GFP fluorescence was observed via microscopy using a GFP filter. The results showed that PM19_00185opt- $\Delta$ TM-GFP was evenly distributed in the cytoplasm of the protoplasts (Fig. 1B).

Using BLAST-P search (NCBI), PM19_00185 did not show any significant similarities with proteins of either other phytoplasmas or other species. To further analyze the potential protein structure of PM19_00185, the amino acid sequence was submitted to PHYRE2 (Protein Homology/Analogy Recognition Engine) for three-dimensional (3D) structure analysis (Kelley et al. 2015). In all, 153 of 200 residues had to be modeled ab initio, indicating that the protein shows no homology to other proteins from databases.

\section{PM19_00185- $\Delta$ TM interacts with several members} of the E2 superfamily of $A$. thaliana in Y2H screens.

To identify plant proteins that could interact with PM19_00185, a Y2H screen using a normalized A. thaliana library (Takara Bio USA, Inc., Mountain View, CA, U.S.A.) fused to the Gal4 activation domain (AD) (expressing plasmid pGADT7) for preys was performed. Based on the result of topology prediction, only the C-terminal noncytoplasmic part of PM19_00185 (amino acids 42 to 200) comes into direct contact with the host cell. Thus, to avoid false-negative results due to nonsoluble protein expression caused by the transmembrane domain, a truncated version of PM19_00185 was produced by deleting the transmembrane domain $(\Delta \mathrm{TM})$ (amino acids 1 to 41 ) and fused to Gal4 binding domain (BD), resulting in the plasmid pGBKT7-PM19_00185- $\triangle T M$, which was used as a bait. In total, $5.5 \times 10^{7}$ clones containing both expressing plasmids were screened for interaction upon resistance to antibiotic aureobasidin A (AbA), expression of $\alpha$-galactosidase, and growth on media lacking histidine and adenine. Approximately 1,000 clones were found to activate all reporter genes. Of these clones, $10 \%$ were randomly selected and the plasmids were isolated and sequenced. The sequence data were analyzed via BLAST in the NCBI database. The results show that all identified genes belong to a superfamily of genes encoding A. thaliana ubiquitin-conjugating enzymes (AtUBC; E2) and can be categorized into three different groups: AtUBC group number III (3\% of screened clones), with AtUBC2; AtUBC group number V (14\%), with AtUBC07 and AtUBC14; and AtUBC group number VI (83\%), with AtUBC08, AtUBC09, and AtUBC10 (nomenclature according to Kraft et al. 2005) (Supplementary Table S1).

Yeast cells are able to take up more than one plasmid during transformation. Therefore, working with library-scale $\mathrm{Y} 2 \mathrm{H}$ screening can lead to false-positive results. To confirm the actual interactions, cotransformations of each candidate prey (pGADT7 plasmids carrying $A D$ - $A t U B C$-fusions were isolated from original positive transformants) with pGBKT7PM19_00185- $\triangle T M$ were conducted. The pGBKT7-empty expressing only $\mathrm{BD}$ was included for a negative control. The cotransformants were screened for resistance to $\mathrm{AbA}$ and
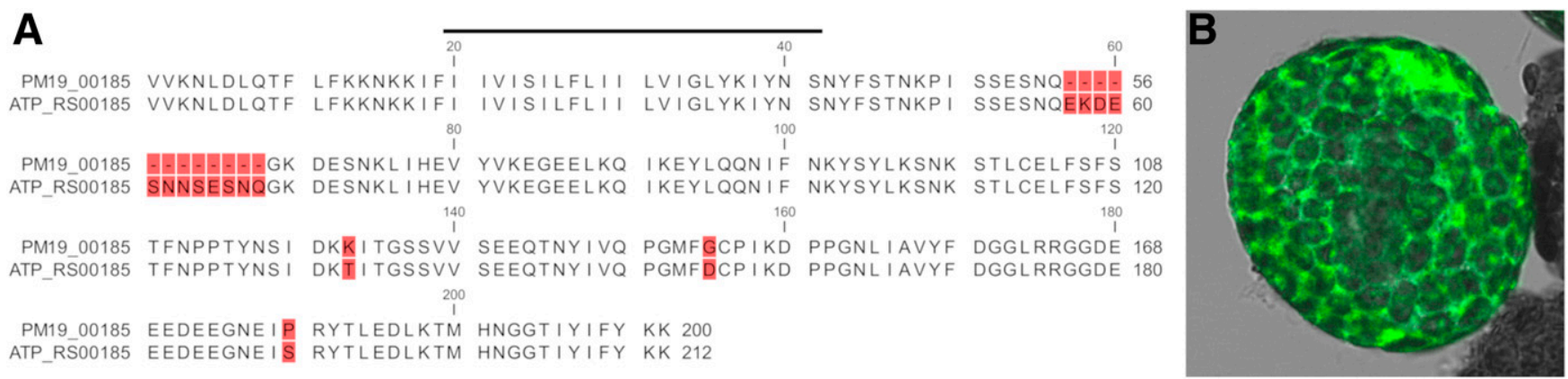

Fig. 1. A, Sequence analysis of PM19 00185 protein. PM19 00185 has a $92 \%$ identity with ATP RS00185 on the amino acid level (differences are shaded). Phobius predictor predicts a transmembrane region from amino acid 20 to 41 in PM19_00185 (black line), with a cytoplasmic N-terminal area (amino acids 1 to 19) and a noncytoplasmic C-terminal area (amino acids 42 to 200). B, Expression of PM19_00185opt- $\Delta$ TM-GFP in Nicotiana benthamiana. Two days after Agrobacterium-mediated infiltration, protoplasts were prepared. Fluorescence microscopy showed that the fusion protein was evenly distributed in the cytoplasm. 
expression of $\alpha$-galactosidase. All combinations of AD-AtUBCfusions and BD-PM19_00185- $\Delta$ TM showed activation of both reporter genes, whereas the combination of $\mathrm{AD}-\mathrm{AtUBC}$-fusions with only BD did not (Fig. 2). Thus, these results confirm our findings in library-scale $\mathrm{Y} 2 \mathrm{H}$ screening.

\section{Recombinant expression of PM19_00185 in Escherichia coli.}

E2 ubiquitin ligases perform the second step of the ubiquitin conjugating cascade and are able to accept ubiquitin from an E1 ubiquitin-activating enzyme that is later transferred to a target protein via an E3 ubiquitin ligase (Glickman and Ciechanover 2002). Thus, E2 binding activity of PM19_00185 could affect the plant ubiquitination system in a way that is beneficial for the pathogen by disturbing or enhancing the enzymatic activity of the E2 protein; or, it could somehow change the substrate specificity.

To further study the possible function of PM19_00185 interaction with $A$. thaliana E2 proteins, we recombinantly expressed PM19_00185 and one of the E2 proteins identified in the Y2H screen, AtUBC09. The expression of PM19_00185$\triangle \mathrm{TM}$ in Escherichia coli led to insoluble protein (data not shown) whereas PM19_00185- $\Delta$ TM was partially soluble when it was expressed as a fusion protein with maltose-binding protein (MBP)-PM19_00185- $\triangle \mathrm{TM}$. However, the MBPPM19_00185- $\triangle$ TM fused protein could not be purified with either amylose resin or anti-MBP bead (data not shown). According to the manufacturer, it is possible that PM19_00185$\triangle \mathrm{TM}$ somehow blocks or distorts the maltose-binding site of MBP, leading to a disturbed affinity to resin and beads. In order to purify the protein, hexa-histidine tags were fused with MBPPM19_00185- $\triangle \mathrm{TM}$ at the $\mathrm{N}$ and $\mathrm{C}$ terminals.

AtUBC09 has the highest consensus sequence within group VI of the E2 superfamily, which was most often found in all positive clones in $\mathrm{Y} 2 \mathrm{H}$ screening. Therefore, it was selected for recombinant expression fused with the hexa-histidine tag at the $\mathrm{N}$ and $\mathrm{C}$ terminals in $E$. coli.

After inducing protein expression with isopropyl- $\beta$-Dthiogalactoside, the expressed proteins were verified by sodium dodecyl sulfate polyacrylamide gel electrophoresis (SDSPAGE) and Western blotting, using anti-His antibodies. Clear protein bands of approximately 63 and $20 \mathrm{kDa}$ for his-MBPPM19_00185- $\triangle \mathrm{TM}$-his and for his-AtUBC09-his, respectively, were detected in both soluble and inclusion body fraction, indicating that the recombinant proteins were partially expressed as soluble proteins (data not shown). The expressed proteins were successful purified under native conditions using $\mathrm{Ni}$ NTA-based purification. Purity of the recombinant proteins was confirmed using SDS-PAGE followed by Coomassie Brilliant Blue staining (Fig. 3A).

\section{PM19_00185 shows E3 ubiquitin ligase activity.}

To confirm the enzymatic activity of the purified AtUBC09, a thioester assay was performed using a ubiquitylation assay kit (Abcam plc, Cambridge, U.K.). In the thioester assay, E1 enzyme activated free ubiquitin by forming a high-energy thiol ester E1-ubiquitin intermediate. In the presence of active E2 enzyme, ubiquitin is transferred to E2, resulting in a second E2-ubiquitin intermediate. The thioester assay was performed according to the manufacturer's protocol using the purified AtUBC09 as E2 enzyme. For a negative control, a reaction without the AtUBC09 was performed. The result of Western blotting using antiubiquitin antibody $(\alpha-\mathrm{Ub})$ to detect ubiquitin-conjugated proteins showed a high-molecular mass smear of polyubiquitinated proteins in the reaction with the purified AtUBC09 that was absent in the negative control, suggesting that the formation of E2-ubiquitin intermediate and, thus, the enzymatic activity of the purified AtUBC09 was confirmed (Fig. 3B).

To study possible effects of PM19_00185 on the enzymatic activity of its interaction partner AtUBC09, the purified hisMBP-PM19_00185- $\Delta$ TM-his was added into the thioester assay of his-AtUBC09-his. No differences were observed comparing the Western blotting result using $\alpha$-Ub of the reactions with and without his-MBP-PM19_00185- $\Delta$ TM-his, suggesting that hisMBP-PM19_00185- $\Delta$ TM-his does not interfere with the formation of E2-ubiquitin intermediates (data not shown).

Therefore, we further investigated the possible function of MBP-PM19_00185- $\triangle$ TM as E3 ligase. E3 ligases recognize target proteins of the ubiquitination pathway and facilitate the transfer of ubiquitin from E2 to these proteins (Glickman and Ciechanover 2002). The thioester assays were performed as described for E2 and anti-MBP antibody ( $\alpha$-MBP) was used for detection in Western blot analysis. The result showed that a high-molecular-mass smear was detected only in the reaction including MBP-PM19_00185- $\triangle \mathrm{TM}$ and AtUBC09 (Fig. 3C). This result suggests that PM19_00185 has an E3 ligase activity and could transfer the ubiquitin chain to a specific target via an E3-ubiquitin intermediate complex.

\section{E3 ubiquitin ligase PM19_00185 is also active with E2} ubiquitin-conjugating enzyme UBC10 of Malus domestica.

A. thaliana is not a natural host plant of ' $\mathrm{Ca}$. P. mali', although it was shown that different plant species share homologs of E2 proteins with a high sequence similarity (Zhou et al. 2017). To confirm that the his-MBP-PM19_00185- $\Delta$ TM-his can also interact with E2 of the natural host, Malus domestica,

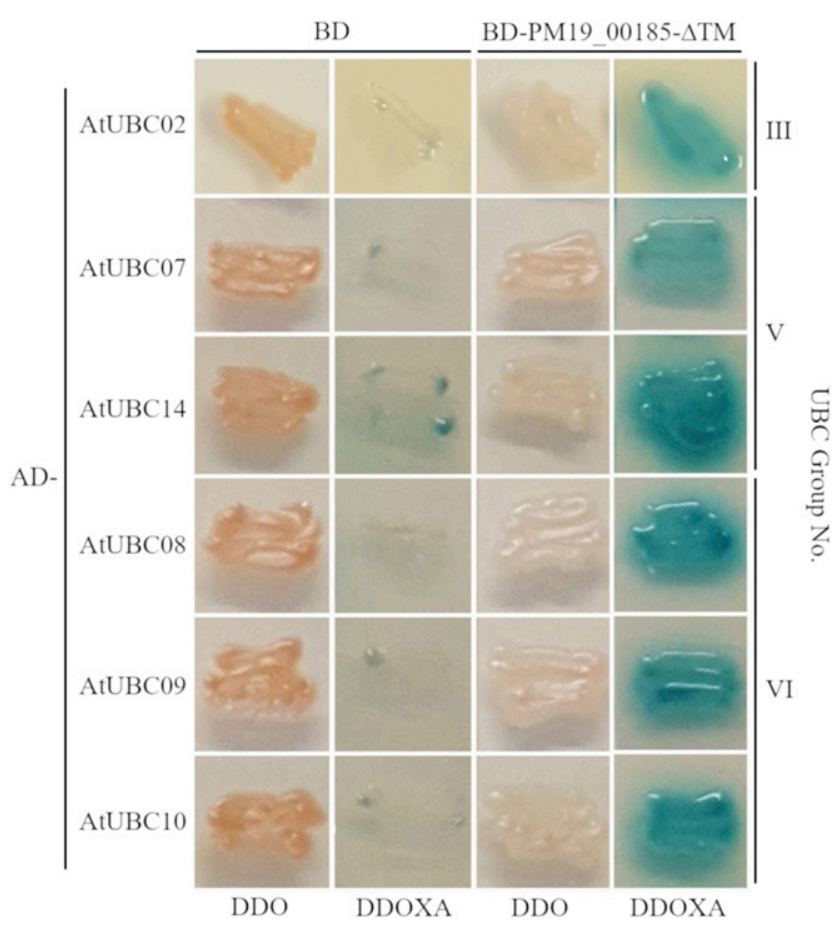

Fig. 2. PM19_00185 binds six ubiquitin-conjugating enzyme (UBC) proteins of Arabidopsis thaliana (AtUBC) belonging to three different groups. A yeast two-hybrid $(\mathrm{Y} 2 \mathrm{H})$ screen was performed using the binding-domain (BD) fused PM19_00185- $\triangle \mathrm{TM}$, resulting in expression plasmid pGBKT7PM19_00185- $\triangle \mathrm{TM}$ and an expression plasmid carrying the activation domain (AD) fused to one of the six AtUBC previously identified in the $\mathrm{Y} 2 \mathrm{H}$. As negative control, empty pGBKT7 expressing only BD was used. Cotransformed yeast cells were patched on double drop-out (DDO) medium to select for the presence of both expressing plasmids and DDO media containing aureobasidin $\mathrm{A}$ and $\mathrm{X}-\alpha-\mathrm{Gal}$ (DDOXA) to select for protein interaction. 


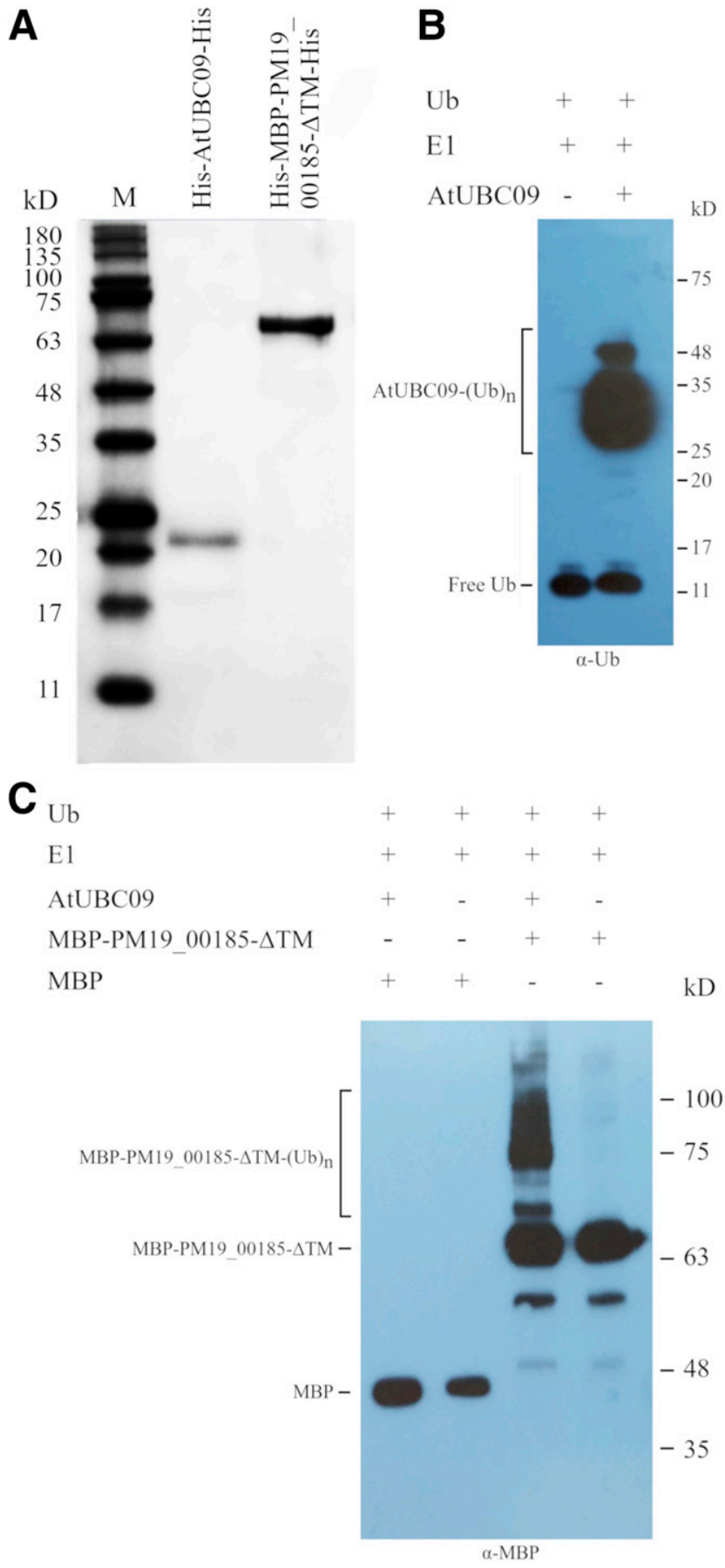

Fig. 3. Recombinant expressed PM19_00185- $\Delta$ TM acts as E3 ubiquitin ligase in the presence of AtUBC09. A, Sodium dodecyl sulfate polyacrylamide gel electrophoresis (SDS-PAGE) analysis of recombinant expressed and purified his-MBP-PM19_00185- $\triangle$ TM-his and his-AtUBC09-his. His-tagged proteins were expressed in Escherichia coli and purified using Ni-NTA-based purification. After separating using SDS-PAGE, the gel was stained with Coomassie brilliant blue. Lane $\mathrm{M}=$ a protein marker. B, In vitro ubiquitination assay confirms the E2 activity of the purified his-AtUBC09-his. Polyubiquitinated forms of his-AtUBC09-his (AtUBC09-[Ub]n) can be detected as a high-molecular-mass smear by Western blotting using antiubiquitin antibody $(\alpha-\mathrm{Ub})$. Reaction without his-AtUBC09-his is used as a negative control. C, In vitro ubiquitination assay suggests E3 ligase activity of PM19_00185. In the presence of E1 and purified his-AtUBC09-his as E2, his-MBP-PM19_00185- $\Delta$ TM-his is polyubiquitinated. Polyubiquitinated forms of his-MBP-PM19_00185- $\Delta$ TM-his (MBP-PM19_00185- $\Delta$ TM$[\mathrm{Ub}] \mathrm{n})$ were detected in Western blotting using anti-maltose-binding protein (MBP) antibody ( $\alpha$-MBP). Reactions without his-AtUBC09-his or only MBP were used as negative controls. All in vitro ubiquitination assays have been repeated at least twice with similar results. an E2 of $M$. domestica was recombinantly expressed and used for an in vitro ubiquitination assay. In this experiment, the MdUBC10 (accession number XP_008376684) was chosen due to its high similarity with AtUBC09 of $97 \%$. The gene was amplified from a full-length cDNA library of Malus $\times$ domestica 'Golden Delicious' (see Material and Methods for details). We recombinantly expressed MdUBC10 as a His-tag fused protein in E. coli as described for his-AtUBC09-his. After NiNTA-based purification, the purity of protein was verified in an SDS-PAGE and Coomassie staining (Fig. 4A).

A ubiquitination assay was performed according to the previous experiments using his-MdUBC10-his for E2 and hisMBP-PM19_00185- $\Delta$ TM-his for E3. The Western blot result using anti-MBP shows high-molecular-mass smears in reactions containing both his-AtUBC09-his and his-MdUBC10-his, indicating E3 ligase enzymatic activity of PM19_00185 in combination with E2 MdUBC10 (Fig. 4B).

\section{Expression of PM19_00185 may suppress basal defense in A. thaliana.}

Targeting the plant ubiquitination system has emerged as a common strategy of plant pathogens. At least two pathogens secret effector proteins that possess an E3 ubiquitin ligase activity: Pseudomonas syringae with AvrPtoB (Abramovitch et al. 2006; Janjusevic et al. 2006) and Xanthomonas campestris with XopL (Singer et al. 2013). In both cases, a suppressing effect on the plant immune system has been reported (Abramovitch and Martin 2005; Rosebrock et al. 2007; Singer et al. 2013). AvrPtoB was shown to suppress immunity-associated programmed cell death (PCD) in the absence of the Pto resistance protein (Abramovitch and Martin 2004).

Phytoplasma cannot be cultivated in vitro. Therefore, to elucidate whether PM19_00185 has a similar influence on the plant immune system, we tested its effect on $P$. syringae infection using a plant immunity assay. It was reported that $P$. syringae pv. tabaci, to which A. thaliana is a nonhost plant, induces A. thaliana NONHOST1 (NHO1) in a flagellindependent manner required for limiting the in planta growth of nonhost Pseudomonas bacteria (Li et al. 2005). It was also shown that coexpression of an effector AvrPtoB promotes the virulence of a nonhost pathogen, $P$. syringae pv. phaseolicola RW60, to A. thaliana and bean (Torres et al. 2006). Therefore, we produced transgenic $A$. thaliana Col-0 expressing PM19_00185opt- $\triangle \mathrm{TM}$ N-terminal fused to Myc-Tag under control of the $35 \mathrm{~S}$ promoter (35S::Myc-PM19_00185opt$\Delta T M)$. Expression of Myc-PM19_00185opt- $\Delta \mathrm{TM}$ was confirmed via Western blot using anti-Myc-antibody (Fig. 5A). The high-molecular-mass smear in the Western blot could indicate ubiquitinated forms of PM19_00185opt- $\Delta$ TM. F2 generation of transgenic $A$. thaliana lines did not show any differences in growth or appearance compared with wild-type Col-0. For the plant immunity assay, leaves of transgenic A. thaliana expressing Myc-PM19_00185opt- $\Delta$ TM and wildtype Col-0 were inoculated with the nonhost pathogen P. syringae pv. tabaci strain ATCC 11527 . After $48 \mathrm{~h}$, infection symptoms were observed in the inoculated plant leaves of transgenic plants whereas they were absent in wild-type leaves (Fig. 5B). Three days after infiltration, the infiltrated leaves were homogenized and centrifuged and the supernatant was serial diluted and plated on King's B agar plates. After $24 \mathrm{~h}$, colonies were counted and CFU were calculated. Log (CFU) of the wild-type sample was $2.08 \pm 0.04$, of transgenic line 1 was $8.13 \pm 0.05$, and of line 2 was $7.78 \pm 0.07$ (Fig. 5C). The result of colony counting clearly shows that $P$. syringae pv. tabaci can grow much higher in transgenic plants expressing PM19_00185opt- $\Delta \mathrm{TM}$ that in wild-type Col-0. The result suggests that PM19_00185opt- $\Delta$ TM could inhibit basal plant 
defense, which would allow the growth of P. syringae pv. tabaci in A. thaliana.

\section{DISCUSSION}

' $\mathrm{Ca}$. P. mali' is the cause of AP, a plant disease that leads to heavy losses in apple production all over Europe. The sequencing

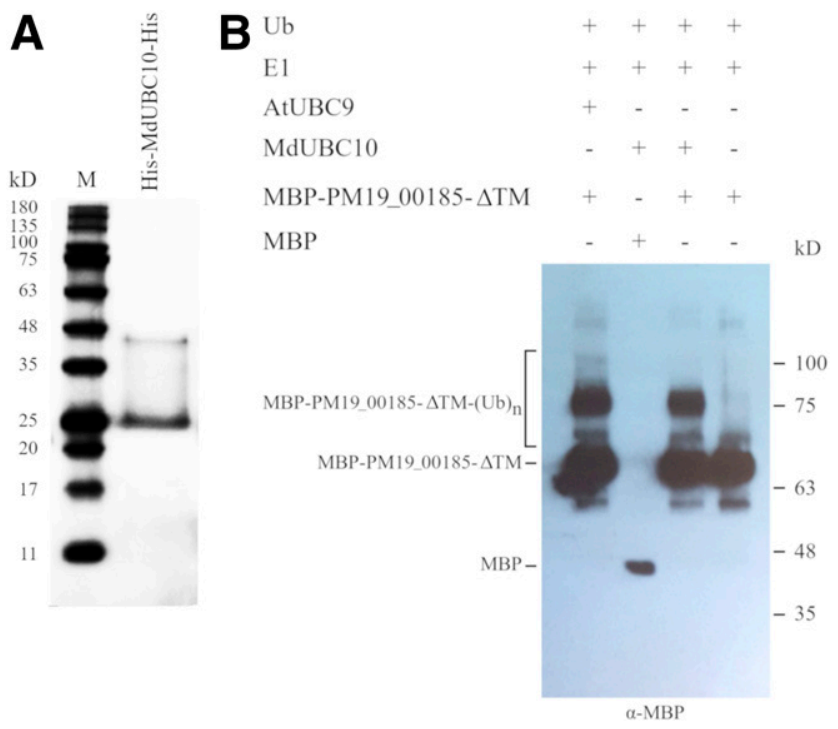

Fig. 4. PM19_00185- $\triangle \mathrm{TM}$ is also active with ubiquitin-conjugating enzyme (UBC)10 of Malus domestica (MdUBC10). A, Sodium dodecyl sulfate polyacrylamide gel electrophoresis (SDS-PAGE) analysis of recombinantly expressed and purified MdUBC10. His-tagged protein was expressed in Escherichia coli and purified using Ni-NTA-based purification. After separating using SDS-PAGE, the gel was stained with Coomassie brilliant blue. Lane $\mathrm{M}=$ a protein marker. $\mathbf{B}$, In vitro ubiquitination assay shows E3 ligase activity of PM19_00185 with MdUBC10. Ub = ubiquitin. In the presence of E1 and purified his-MdUBC10-his, his-MBP-PM19_00185- $\triangle$ TM-his is polyubiquitinated. Polyubiquitinated forms of his-MBP-PM19_00185- $\Delta$ TM-his (MBP-PM19_00185- $\Delta$ TM-[Ub]n) were detected using anti-maltose-binding protein (MBP) antibody ( $\alpha$-MBP). Reactions without his-MdUBC10-his or only MBP were used as negative controls and the reaction with his-AtUBC09his as a positive control. All in vitro ubiquitination assays have been repeated at least twice, with similar results. of the complete genome of ' $\mathrm{Ca}$. P mali' strain AT (Kube et al. 2008) gave valuable insight into this thus-far poorly characterized pathogen. We used Phobius prediction-based (Käll et al. 2004) sequence data to select 23 proteins that contain a transmembrane region and, thus, are potentially located outside of the phytoplasma cell. Because these proteins would come in direct contact with the host cell, we hypothesize that some of these proteins could directly interact with host plant proteins during the infection. Thus, we performed $\mathrm{Y} 2 \mathrm{H}$ screenings with plant libraries to search for new pathogen-host protein interactions. In a similar approach, a list of AY-WB phytoplasma proteins containing an N-terminal SP sequence that are secreted in the plant cell was used to find the effector protein SAP11, which interacts with CIN-related TCP transcription factors of the host plant (Bai et al. 2009).

In this work, we present the function of a previously undescribed protein of ' $C a$. P. mali' strain PM19, named PM19_00185. Its corresponding protein in ' $\mathrm{Ca}$. P. mali' strain AT is ATP_RS00185, which was annotated as a hypothetical protein (Kube et al. 2008). Sequence and structure analysis did not show any similarities to other proteins or known domains. We showed that amino acids 42 to 200 of this protein localize in the cytoplasm of plant cells when fused to GFP and transiently expressed in $N$. benthamiana. Using the $\mathrm{Y} 2 \mathrm{H}$ approach, we found that PM19_00185 interacted with at least six E2 enzymes of three different UBC groups of A. thaliana. Since phytoplasmas reside in the phloem of their host plants and members of the proteasomal degradation pathway have been identified in the phloem of plants (Giavalisco et al. 2006; Lin et al. 2009) and might be fully active (Ostendorp et al. 2017), PM19_00185 could be active in the phloem or could be transported elsewhere after being released from the phytoplasma. However, further experiments are necessary to confirm either of these theories.

The A. thaliana genome was predicted to encode 37 E2 proteins that were divided into 16 groups based on their identity to each other (Kraft et al. 2005). Within one group, E2 proteins can show a high similarity to each other (Kraft et al. 2005). The hierarchy of the ubiquitin system is not of a linear or pyramid structure but is more a complex network of overlapping interactions. Most E2s interact with several E3s and E3s can often interact with more than one E2 (Glickman and Ciechanover 2002). Thus, it is no surprise that PM19_00185 interacts with
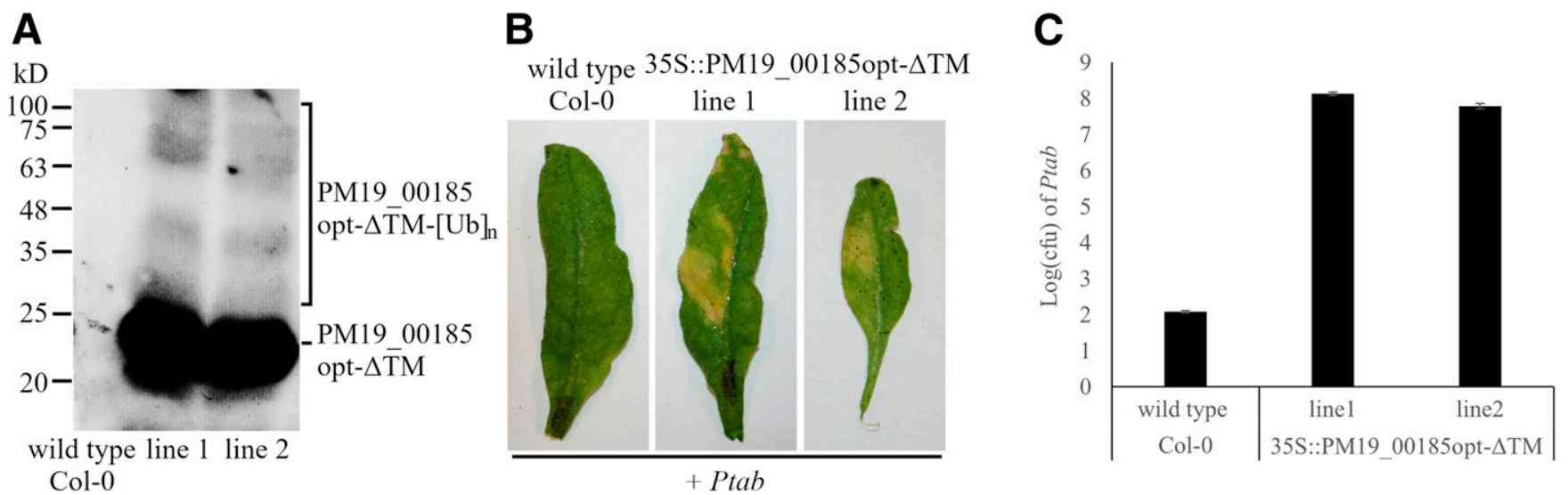

Fig. 5. Pseudomonas syringae pv. tabaci (Ptab) can grow in transgenic Arabidopsis thaliana plants expressing PM19 00185. A, Western blot of protein extracts of $A$. thaliana Col-0 wild-type and transgenic plant lines 35S::Myc-PM19_00185opt- $\Delta$ TM. Myc-PM19_00185opt- $\Delta$ TM expression was detected in the transgenic lines using Anti-Myc-antibody. High-molecular-mass smear could indicate ubiquitinated forms of PM19_00185opt- $\Delta$ TM [UB]n). B, Plant immunity assay. Wild-type Col-0 and 35S::Myc-PM19_00185opt- $\Delta$ TM leaves were inoculated with $P$. syringae pv. tabaci. Infection symptoms were observed $48 \mathrm{~h}$ after the $P$. syringae pv. tabaci inoculation. The experiment was repeated independently three times with at least three replicates each time with similar results. C, In planta growth of $P$. syringae pv. tabaci in Arabidopsis Col-0 expressing PM19_00185opt- $\Delta$ TM was higher than that of the wild type. Columns show means and standard deviation of CFU of three independent experiments. 
different groups of $\mathrm{E} 2$ in the $\mathrm{Y} 2 \mathrm{H}$ screening. In our experiments, we found that AtUBC02 of group III; AtUBC07 and AtUBC14 of group V; and AtUBC08, AtUBC09, and AtUBC10 of group VI interacted with PM19_00185. However, we only analyzed $10 \%$ of positive clones and, due to the high similarity within groups, it is possible that PM19_00185 could interact with more $\mathrm{E} 2$ proteins of $A$. thaliana. Because $\mathrm{Y} 2 \mathrm{H}$ is not an in vivo method, it is also possible that PM19_00185 could interact with proteins that need specific conditions only present in plant cells or require binding of additional proteins not present during the $\mathrm{Y} 2 \mathrm{H}$ screening study. Thus, we might not have detected all possible functions of PM19_00185.

Nevertheless, our finding gives valuable insight into a potential function of PM19_00185 and, therefore, we further analyzed the interaction in terms of the effect of PM19_00185 on the enzymatic activity of the A. thaliana ubiquitination system. PM19_00185 fused to MBP for increasing the solubility and one of the most abundant E2 protein found in $\mathrm{Y} 2 \mathrm{H}$, AtUBC09, were recombinantly expressed and purified. In a ubiquitination assay, we detected a high-molecular-mass smear in reactions including MBP-PM19_00185- $\triangle \mathrm{TM}$ and AtUBC09. Because the smear is detectable by using $\alpha$-MBP, it indicates the formation of polyubiquitinated forms of MBPPM19_00185- $\triangle$ TM. E3 ligases are crucial for the transfer of the activated ubiquitin to a substrate. The activated ubiquitin is either directly transferred to the E3-bound substrate or an E3ubiquitin intermediate is formed before the ubiquitin is transferred (Glickman and Ciechanover 2002). Although no other additional $\mathrm{E} 3$ protein was added to the reactions, the ubiquitin chains were transferred from E2 protein AtUBC09 to HisMBP-PM19_00185- $\Delta$ TM-His. This strongly suggests that PM19_00185 has an E3 ligase activity.

Furthermore, different plant species can share homologs of E2 proteins that have a high sequence similarity (Zhou et al. 2017). Therefore, it is likely that PM19_00185 is also active with several E2 enzymes of the natural host of ' $\mathrm{Ca}$. P. mali', M. domestica. We demonstrated that PM19_00185, indeed, is active with E2 MdUBC10. This is not the first time that the effector proteins of plant pathogens with E3 ligase activity were reported. AvrPtoB of $P$. syringae is polyubiquitinated specifically in the presence of tomato (Solanum lycopersicum) E2 (SIUBC) group III, which is closely related to AtUBC group VI (Zhou et al. 2017). It was also shown that AvrPtoB and XopL, an E3 ligase protein of $X$. campestris, were active with E2 of AtUBC group VI (Abramovitch et al. 2006; Singer et al. 2013), which is the major group of UBC enzymes that interact with phytoplasma PM19_00185. These findings imply some similarities of mode of action of these proteins.

Ubiquitin conjugation is a multistep reaction involving three enzymes: E1 (ubiquitin-activating enzyme), E2 (UBC), and E3 (ubiquitin ligase) (Glickman and Ciechanover 2002). In an ATP-dependent reaction, E1 activates ubiquitin, resulting in a thioester-linked ubiquitin. The thioester-linked ubiquitin is transferred to E2, resulting in a high-energy intermediate. E3 enzymes specifically recognize a substrate and facilitate the transfer of ubiquitin to the substrate by tethering them in close proximity (RING finger or U-Box E3s) (Vierstra 2009) or a further intermediate is formed where the thioester-linked ubiquitin is bound to E3 before it is transferred to the substrate (HECT domain E3s) (Glickman and Ciechanover 2002). In the in vitro ubiquitination assay, the PM19_00185 was polyubiquitinated in the presence of E1 and E2 without other additional E3, suggesting that the mechanism of action of PM19_00185 is likely to be the latter. E3 proteins are classified into four main subfamilies depending on their structural features and mechanism of action (Duplan and Rivas 2014). The structural analysis of XopL revealed a unique folding for amino acids 474 to 660 that lacked any similarities to other E3 ligases (Singer et al. 2013); thus, it presents a new group of E3. Because PM19_00185 does not contain any signature structure of E3 and the 3D-computed prediction gives no identity to any known E3, it is possible that PM19_00185 could also represent a new group of E3 ligases. A structure analysis of PM19_00185 would be required to further illustrate features of this protein.

In recent years, the plant ubiquitination system has emerged as a common target of plant pathogen effector proteins (Banfield 2015). Several E3 ligase proteins have been identified as modulators of the first step of pathogen recognition by plant cells (Duplan and Rivas 2014). For plant bacteria pathogens, two proteins of type III secreted effectors were identified to have an E3 ligase activity and are active with plant E2 enzymes (Janjusevic et al. 2006; Singer et al. 2013). The P. syringae effector protein AvrPtoB contains a C-terminal domain with E3 ubiquitin ligase activity (Abramovitch et al. 2006; Janjusevic et al. 2006) and disrupts plant immunity by specifically ubiquitinating the host kinase Fen (Rosebrock et al. 2007). In the absence of the Pto resistance protein, AvrPtoB suppresses PCD and immunity in tomato (Abramovitch and Martin 2005). The E3 ligase activity of XopL of $X$. campestris is located in its $\mathrm{C}$ terminal XL domain and was discussed to suppress the plant immune system by ubiquitination of a thus-far-unknown plant substrate (Singer et al. 2013).

In this work, we show that PM19_00185- $\Delta$ TM has E3 ligase activity and could suppress basal plant defense in A. thaliana, which allows growth of the nonhost pathogen $P$. syringae pv. tabaci strain ATCC 11527. Although PM19_00185- $\Delta$ TM has E3 ligase activity similar to AvrPtoB, we cannot postulate the same mechanism of plant immunity as observed with AvrPtoB. Therefore, the elucidation of the actual mechanism of PM19_00185 E3 ligase on plant immunity requires further studies. Nevertheless, our results strongly suggest that PM19_00185 could suppress basal defense mechanisms during ' $C a$. P. mali' infection, leading to a higher susceptibility of the plant toward the pathogen.

In contrast to the phytoplasma effector SAP54, which promotes degradation of MADS-box transcription factors by binding RAD23, a plant protein, and shuttling of the substrate to 26S proteasome (MacLean et al. 2011, 2014), the PM19_00185 has E3 ligase activity and probably binds to target proteins prior to loading to the ubiquitin-proteasome system. Nevertheless, our findings suggest that phytoplasmas may affect the ubiquitin pathway and highjack it in a way that increases the pathogen fitness, To the extent of our knowledge, this is the first study showing that a phytoplasma effector protein has an activity similar to E3 ligase proteins and might suppress basal defense. To fully understand how PM19_00185 affects the plant host ubiquitin system and to elucidate the suppressing activity of the protein on the plant basal defense, further investigations are required.

\section{MATERIALS AND METHODS}

\section{Origin of PM19_00185 DNA.}

PM19_00185 (GenBank: MK552110) was amplified from total DNA isolated from ' $\mathrm{Ca}$. P. mali' strain PM19 that was preciously transmitted from field-collected $C$. picta to healthy test plants of Malus $\times$ domestica (Jarausch et al. 2011). Total nucleic acids from plants tissue were extracted with a modified cetyltrimethylammonium bromide-based protocol described elsewhere (Jarausch et al. 2011). Primers for gene amplification were designed using genomic data from the corresponding gene of 'Ca. P. mali' strain AT ATP_RSO0185 (Kube et al. 2008): 5'-GTGGTAAAAAATTTGGATTTACAAACA-3' and 5' CTTTTTTATAAAAAATATAAATAGTTCCTCCA-3'. 
Transient protein expression in planta and protoplast preparation.

Agroinfiltration was performed as previously described (Schöb et al. 1997). In short, a single colony of Agrobacterium tumefaciens strain ATHV transformed with $35 \mathrm{~S}:$ : PM19_00185opt- $\triangle T M-G F P$ was grown at $28^{\circ} \mathrm{C}$. Bacteria were centrifuged, resuspended in induction media (bacterial growth medium substituted with $10 \mathrm{mM}$ morpholineethanesulfonic acid [MES], $2 \mathrm{mM} \mathrm{MgSO}$, and $0.05 \mathrm{mM}$ acetosyringone) and grown overnight at $28^{\circ} \mathrm{C}$. After centrifuging, the cell pellet was resuspended to an optical density at $600 \mathrm{~nm}\left(\mathrm{OD}_{600}\right)$ of 2.4 in infection media (half-strength Murashige and Skoog [1/2 MS] media substituted with $10 \mathrm{mM}$ MES, $2 \%$ sucrose, and $0.2 \mathrm{mM}$ acetosyringone) and incubated at room temperature for at least $2 \mathrm{~h}$. The bacteria were infiltrated into leaves of $N$. benthamiana plants using a $1-\mathrm{ml}$ needleless syringe.

For protoplast preparation, two leaves were collected 2 days after agroinfiltration and cut in small strips of approximately $1 \mathrm{~mm}$. The strips were placed in enzyme solution for cell wall maceration $(1 / 2 \mathrm{MS}$ media at $\mathrm{pH} 5.8$ substituted with $0.4 \mathrm{M}$ sucrose, $1 \%$ Cellulase Onozuka R-10, and 0.2\% Macerozyme R-10 [both Serva, Heidelberg, Germany]), infiltrated by applying a short vacuum, and then incubated for $2 \mathrm{~h}$ on a gyratory shaker $(20 \mathrm{rpm})$. Protoplasts were carefully collected by centrifuging at $100 \times g$ and examined using a Zeiss Observer Z1 with the LSM510 confocal laser-scanning head.

\section{Y2H screening.}

$\mathrm{Y} 2 \mathrm{H}$ screening was performed using the Matchmaker Gold Yeast Two-Hybrid System (Takara Bio USA, Inc.). For bait, an N-terminal deletion mutant of PM19_00185 (amino acids 41 to $200)$ without transmembrane region $(\Delta \mathrm{TM})$ was amplified using primers introducing restriction sites $\mathrm{NcoI}$ and BamHI at the $5^{\prime}$ and $3^{\prime}$ ends of the gene, respectively $\left(5^{\prime}\right.$-GCCATGGC CAGTAACTATTTTTCG-3' and 5'-CGGATCCTTTTTTATA AAAAATAT- $3^{\prime}$ ). The PCR products were digested and cloned into pGBKT7 vector (TRP1 nutritional marker; Takara Bio USA, Inc.) for BD fusion. pGBKT7-PM19_00185- $\triangle T M$ was transformed into yeast strain Y2H Gold (Mat $\alpha)$ and tested for toxicity and autoactivation of reporter genes. For prey, the Mate\&Plate Library-Universal Arabidopsis (normalized) (Takara Bio USA, Inc.) was used. It contains cDNA derived from 11 Arabidopsis thaliana tissues, cloned into pGADT7 vector (LEU2) for AD fusion, and transformed into yeast strain Y187 (Mat $\alpha)$.

$\mathrm{Y} 2 \mathrm{H}$ screening was performed according to the manufacturer's protocol. This protocol uses four different reporter genes to detect protein interactions. AUR1-C confers strong resistance to AbA and MEL1 encodes $\alpha$-galactosidase. When the substrate $\mathrm{X}$ - $\alpha$-gal (5-bromo-4-chloro-3-indolyl- $\alpha$-D-galactopyranoside) is added to the media, positive colonies turn blue. HIS3 and ADE2 allow cells to grow on -His and -Ade minimal medium. Different yeast drop-out media were purchased from Sigma-Aldrich Inc. (St. Louis) and prepared according to the manufacturer's instructions. X- $\alpha$-gal at $40 \mu \mathrm{g} / \mathrm{ml}$ (Iris Biotech GmbH, Marktredwitz, Germany) and AbA at $200 \mathrm{ng} / \mathrm{ml}$ (Takara Bio USA, Inc.) were added when required.

In short, a concentrated culture of yeast strain Y2H Gold (Mat $\alpha)$ transformed with pGBKT7-PM19_00185- $\triangle T M$ was mated with $1 \mathrm{ml}$ of Arabidopsis Mate\&Plate library. Numbers of screened cloned were calculated by growth on -Leu-Trp double drop-out (DDO) plates that selects for mated cells. The rest of the mated culture was plated on 60 DDO plates $(150 \mathrm{~mm}$ in diameter) containing X- $\alpha-\mathrm{Gal}$ and AbA (DDOXA). Blue colonies were patched out on the higher stringency quadruple dropout medium QDOXA (DDOXA with additional -His and -Ade).
QDOXA-positive colonies were analyzed by colony PCR using pGADT7-specific primers 5'T7 (5'-TAATACGACTCA CTATAGG-3') and $3^{\prime}$ AD (5'-AGATGGTGCACGATGCA CAG- $\left.3^{\prime}\right)$. PCR products were purified and sent for sequencing using 5'T7 primer (StarSEQ GmbH, Mainz, Germany).

To confirm the results, pGADT7 plasmids were extracted from positive colonies and cotransformed with pGBKT7PM19_00185- $\triangle T M$ and an empty pGBKT7 for a negative control in yeast strain $\mathrm{Y} 2 \mathrm{H}$ Gold. To test the protein interaction, colonies were patched on DDOXA plates.

\section{Expression and purification of His-AtUBC9-His and His-MdUBC10-His.}

AtUBC09 gene was obtained from a positive clone found in $\mathrm{Y} 2 \mathrm{H}$ screen. MdUBC10 gene was amplified from a full-length cDNA library of Malus $\times$ domestica 'Golden Delicious' that was kindly provided by Fondazione Edmund Mach, Italy, using primer 5'-CATGGCGTCCAAGCGGATC-3' and 5'CTTAGCCCATGGCGTATTTC-3' and cloned into pET28a+ expression vector (Novagen, Madison, WI, U.S.A.) fused with His-tag.

His-tagged AtUBC09 and MdUBC10 were expressed in $E$. coli strain BL21+ and purified according to the protocol of Kraft et al. (2005). Transformed cells were grown at $37^{\circ} \mathrm{C}$ in $500 \mathrm{ml}$ of $2 \mathrm{TY}(1.6 \%$ tryptone, $1 \%$ yeast extract, $0.5 \% \mathrm{NaCl})$ broth to an $\mathrm{OD}_{600}$ of approximately 0.5 . Expression of protein was induced with $1.0 \mathrm{mM}$ isopropyl $\beta$-D-thiogalactopyranoside (Iris Biotech $\mathrm{GmbH}$, Marktredwitz, Germany) for $3 \mathrm{~h}$ at $37^{\circ} \mathrm{C}$ or overnight at $14^{\circ} \mathrm{C}$. Cells were harvested by centrifugation and the proteins were extracted with a lysis buffer containing $25 \mathrm{mM}$ Tris-HCL (pH 7.5), $500 \mathrm{mM} \mathrm{NaCl}, 0.01 \%$ Triton $\mathrm{X}-100$, and $5 \mathrm{mM}$ imidazole.

For protein purification, Protino Ni-NTA Agarose (MachereyNagel GmbH \& Co. KG, Düren, Germany) was added to the rest of the cleared lysate and the mixture was incubated for $1 \mathrm{~h}$, rotating at room temperature. Beads were washed with wash buffer containing $25 \mathrm{mM}$ Tris-HCL (pH 7.5), $300 \mathrm{mM} \mathrm{NaCl}$, $0.01 \%$ Triton $\mathrm{X}-100$, and $5 \mathrm{mM}$ imidazole. Proteins were eluted with $150 \mu$ of elution buffer containing $25 \mathrm{mM}$ TrisHCL (pH 7.5), $150 \mathrm{mM} \mathrm{NaCl}, 0.01 \%$ Triton X-100, and $300 \mathrm{mM}$ imidazole. Protein was stored at $4^{\circ} \mathrm{C}$.

\section{Expression and purification of His-MBP-PM19_00185- $\Delta$ TM-His.}

PM19_00185- $\triangle T M$ was cloned into pMAL expression vector (NEB, Ipswich, MA, U.S.A.) fused with MBP. Thereafter the $M B P-P M-00185-\triangle T M$ was subcloned into a pET28a+ vector fused with His-tags at the $\mathrm{N}$ and $\mathrm{C}$ terminus. The fused protein was expressed and purified as described for His-AtUBC9-His and His-MdUBC10-His, with an additional washing step using buffer containing $25 \mathrm{mM}$ Tris-HCL (pH 7.5), $150 \mathrm{mM} \mathrm{NaCl}$, $0.01 \%$ Triton X-100, and $100 \mathrm{mM}$ imidazole.

The purity of all recombinant proteins was verified in SDSPAGE and stained with Coomassie Brilliant Blue.

\section{Ubiquitination assay.}

In vitro ubiquitination assays were performed using the Ubiquitylation Assay Kit (Abcam plc) according to the manufacturer's protocol.

To determine E2 activity of recombinantly expressed E2 proteins, His-AtUBC9-His, and His-MdUBC10-His, a ubiquitination assay was performed in $50 \mu \mathrm{l}$ of reaction containing $100 \mathrm{nM}$ E1 provided in the kit and $2.5 \mu \mathrm{g}$ of purified E2, $1 \times$ ubiquitinylation buffer, $5 \mathrm{mM} \mathrm{Mg}$-ATP, $2.5 \mu \mathrm{M}$ Ubiquitin (all contained in the kit), and an additional $1 \mathrm{U}$ of IPP (Thermo Fischer Scientific, Waltham, MA, U.S.A.) and $1 \mathrm{mM}$ dithiothreitol (Roche Diagnostics GmbH, Mannheim, Germany). For 
a negative control, the same reaction was performed without the purified E2. The reaction was incubated at $37^{\circ} \mathrm{C}$ for $60 \mathrm{~min}$, mixed with $50 \mu$ of $2 \times$ loading dye provided in the kit. The reaction was either directly analyzed or stored at $-20^{\circ} \mathrm{C}$. For analysis, $20 \mu \mathrm{l}$ of the mixture was separated by SDS-PAGE and transferred onto polyvinylidene diflouride (PVDF) membrane. The membrane was blocked with $3 \%$ bovine serum albumen (BSA) in phosphate-buffered saline (PBS) buffer and ubiquitinconjugated proteins were detected using the anti-ubiquitin antibody provided in the kit, followed by antimouse-conjugated peroxidase (Sigma-Aldrich Inc.).

The E3 activity of His-MBP-PM19_RS00185- $\Delta$ TM-His was determined as described for E2 activity determination, with an additional 0.5 to $1 \mu \mathrm{g}$ of recombinant expressed HisMBP-PM19_00185- $\Delta$ TM-His. Negative controls were performed without E2 enzyme or with MBP. The reaction $(10 \mu \mathrm{l})$ was separated by SDS-PAGE and transferred on PVDF. The membrane was blocked with $3 \%$ skim milk in PBS and proteins were detected using anti-MBP-antibody (NEB) followed by antimouse-conjugated peroxidase (SigmaAldrich Inc.)

\section{Generation and analysis of transgenic $A$. thaliana lines.}

Transgenic $A$. thaliana lines were generated via floral dip, as previously described (Clough and Bent 1998). In short, a single colony of Agrobacterium tumefaciens strain GV3101 transformed with 35S::Myc-PM19_00185opt- $\triangle T M$ was grown overnight at $28^{\circ} \mathrm{C}$. Bacteria were centrifuged and resuspended in infection media (1/2 MS media substituted with $10 \mathrm{mM}$ MES, $5 \%$ sucrose, $0.2 \mathrm{mM}$ acetosyringone, and $0.05 \%$ Silwet L-77) and incubated at room temperature for at least $2 \mathrm{~h}$. Flowers of Arabidopsis thaliana plants were submerged in a bacterial suspension for $30 \mathrm{~s}$. Plants were kept in a dark and humid area for 2 days and were then grown in a greenhouse until seed could be collected.

The F1 generation of transgenic plants was checked for protein expression using Western blot. A small leaf was ground in $200 \mu \mathrm{l}$ of extraction buffer $(50 \mathrm{mM}$ sodium phosphate $[\mathrm{pH}$ 7.0], $10 \mathrm{mM}$ Triton X-100, $10 \mathrm{mM}$ N-lauroylsarcosine, and $1 \mathrm{mM}$ mercaptoethanol). Each extract $(20 \mu \mathrm{l})$ was separated by SDS-PAGE and transferred onto PVDF. The membrane was blocked with 3\% BSA in PBS and proteins were detected using Anti-Myc-antibody (provided by U. Conrad, IPK, Gatersleben, Germany) followed by antimouse-conjugated peroxidase (SigmaAldrich Inc.).

\section{Plant immunity assay.}

The plant immunity assay was performed as described elsewhere (Torres et al. 2006). In short, leaves of wild-type and transgenic $A$. thaliana expressing Myc-PM19_00185opt- $\triangle T M$ were inoculated with $P$. syringae pv. tabaci strain ATCC 11527. The infectious symptoms were observed $48 \mathrm{~h}$ after $P$. syringae pv. tabaci infiltration.

Growth of $P$. syringae pv. tabaci in wild-type and transgenic A. thaliana plants was evaluated by using a syringe to infiltrate the plant leaves with a $P$. syringae pv. tabaci solution (OD of 0.2 ). At 3 days after inoculation, the inoculated leaves were extracted with King's B medium (Carl Roth, Karlsruhe, Germany) and briefly centrifuged. The supernatant was serial diluted, plated on King's B medium agar plates, and incubated at $26^{\circ} \mathrm{C}$. The colonies were counted and CFU were evaluated. The experiment was conducted three times.

\section{ACKNOWLEDGMENTS}

We thank W. Jarausch for providing DNA extract from ' $\mathrm{Ca}$. P. mali' strain PM19; M. Kube for the support in bioinformatic analysis; and
Fondazione Edmund Mach, Italy, for providing the full-length cDNA library of Malus $\times$ domestica 'Golden Delicious'.

\section{LITERATURE CITED}

Abramovitch, R. B., Janjusevic, R., Stebbins, C. E., and Martin, G. B. 2006. Type III effector AvrPtoB requires intrinsic E3 ubiquitin ligase activity to suppress plant cell death and immunity. Proc. Natl. Acad. Sci. U.S.A 103:2851-2856.

Abramovitch, R. B., and Martin, G. B. 2004. Strategies used by bacterial pathogens to suppress plant defenses. Curr. Opin. Plant Biol. 7:356-364.

Abramovitch, R. B., and Martin, G. B. 2005. AvrPtoB: A bacterial type III effector that both elicits and suppresses programmed cell death associated with plant immunity. FEMS Microbiol. Lett. 245:1-8.

Bai, X., Correa, V. R., Toruño, T. Y., Ammar, E.-D., Kamoun, S., and Hogenhout, S. A. 2009. AY-WB phytoplasma secretes a protein that targets plant cell nuclei. Mol. Plant-Microbe Interact. 22:18-30.

Banfield, M. J. 2015. Perturbation of host ubiquitin systems by plant pathogen/pest effector proteins. Cell. Microbiol. 17:18-25.

Bendtsen, J. D., Jensen, L. J., Blom, N., Heijne, G. von, and Brunak, S. 2004. Feature-based prediction of non-classical and leaderless protein secretion. Protein Eng. Des. Sel. 17:349-356.

Bertaccini, A. 2007. Phytoplasmas: Diversity, taxonomy, and epidemiology. Front. Biosci. 12:673-689.

Clough, S. J., and Bent, A. F. 1998. Floral dip: A simplified method for Agrobacterium-mediated transformation of Arabidopsis thaliana. Plan J. 16:735-743.

Duplan, V., and Rivas, S. 2014. E3 ubiquitin-ligases and their target proteins during the regulation of plant innate immunity. Front. Plant Sci. $5: 42$

Giavalisco, P., Kapitza, K., Kolasa, A., Buhtz, A., and Kehr, J. 2006. Towards the proteome of Brassica napus phloem sap. Proteomics 6: 896-909.

Glickman, M. H., and Ciechanover, A. 2002. The ubiquitin-proteasome proteolytic pathway: Destruction for the sake of construction. Physiol Rev. 82:373-428.

Janik, K., Mithöfer, A., Raffeiner, M., Stellmach, H., Hause, B., and Schlink, K. 2017. An effector of apple proliferation phytoplasma targets TCP transcription factors-a generalized virulence strategy of phytoplasma? Mol. Plant Pathol. 18:435-442.

Janjusevic, R., Abramovitch, R. B., Martin, G. B., and Stebbins, C. E. 2006 A bacterial inhibitor of host programmed cell death defenses is an E3 ubiquitin ligase. Science 311:222-226.

Jarausch, B., Schwind, N., Fuchs, A., and Jarausch, W. 2011. Characteristics of the spread of apple proliferation by its vector Cacopsylla picta. Phytopathology 101:1471-1480.

Käll, L., Krogh, A., and Sonnhammer, E. L. L. 2004. A combined transmembrane topology and signal peptide prediction method. J. Mol. Biol. 338:1027-1036.

Kelley, L. A., Mezulis, S., Yates, C. M., Wass, M. N., and Sternberg, M. J. E. 2015. The Phyre 2 web portal for protein modeling, prediction and analysis. Nat. Protoc. 10:845-858.

Kraft, E., Stone, S. L., Ma, L., Su, N., Gao, Y., Lau, O.-S., Deng, X.-W., and Callis, J. 2005. Genome analysis and functional characterization of the E2 and RING-type E3 ligase ubiquitination enzymes of Arabidopsis. Plant Physiol. 139:1597-1611.

Kube, M., Schneider, B., Kuhl, H., Dandekar, T., Heitmann, K., Migdoll, A. M., Reinhardt, R., and Seemüller, E. 2008. The linear chromosome of the plant-pathogenic mycoplasma 'Candidatus Phytoplasma mali'. BMC Genomics 9: Article 306.

Li, X., Lin, H., Zhang, W., Zou, Y., Zhang, J., Tang, X., and Zhou, J.-M. 2005. Flagellin induces innate immunity in nonhost interactions that is suppressed by Pseudomonas syringae effectors. Proc. Natl. Acad. Sci. U.S.A. 102:12990-12995.

Lin, M.-K., Lee, Y.-J., Lough, T. J., Phinney, B. S., and Lucas, W. J. 2009. Analysis of the pumpkin phloem proteome provides insights into angiosperm sieve tube function. Mol. Cell. Proteomics 8:343-356.

MacLean, A. M., Orlovskis, Z., Kowitwanich, K., Zdziarska, A. M., Angenent, G. C., Immink, R. G. H., and Hogenhout, S. A. 2014 Phytoplasma effector SAP54 hijacks plant reproduction by degrading MADS-box proteins and promotes insect colonization in a RAD23dependent manner. PLoS Biol. 12:e1001835.

MacLean, A. M., Sugio, A., Makarova, O. V., Findlay, K. C., Grieve, V. M., Tóth, R., Nicolaisen, M., and Hogenhout, S. A. 2011. Phytoplasma effector SAP54 induces indeterminate leaf-like flower development in Arabidopsis plants. Plant Physiol. 157:831-841. 
Ostendorp, A., Pahlow, S., Krüßel, L., Hanhart, P., Garbe, M. Y., Deke, J., Giavalisco, P., and Kehr, J. 2017. Functional analysis of Brassica napus phloem protein and ribonucleoprotein complexes. New Phytol. 214:1188-1197.

Rosebrock, T. R., Zeng, L., Brady, J. J., Abramovitch, R. B., Xiao, F., and Martin, G. B. 2007. A bacterial E3 ubiquitin ligase targets a host protein kinase to disrupt plant immunity. Nature 448:370-374.

Schöb, H., Kunz, C., and Meins, F. 1997. Silencing of transgenes introduced into leaves by agroinfiltration: A simple, rapid method for investigating sequence requirements for gene silencing. Mol. Gen. Genet. 256: 581-585.

Singer, A. U., Schulze, S., Skarina, T., Xu, X., Cui, H., Eschen-Lippold, L., Egler, M., Srikumar, T., Raught, B., Lee, J., Scheel, D., Savchenko, A., and Bonas, U. 2013. A pathogen type III effector with a novel E3 ubiquitin ligase architecture. PLoS Pathog. 9:e1003121.

Sugio, A., Kingdom, H. N., MacLean, A. M., Grieve, V. M., and Hogenhout, S. A. 2011a. Phytoplasma protein effector SAP11 enhances insect vector reproduction by manipulating plant development and defense hormone biosynthesis. Proc. Natl. Acad. Sci. U.S.A. 108: E1254-E1263.

Sugio, A., MacLean, A. M., Kingdom, H. N., Grieve, V. M., Manimekalai, R., and Hogenhout, S. A. 2011b. Diverse targets of phytoplasma effectors: From plant development to defense against insects. Annu. Rev. Phytopathol. 49:175-195.

Torres, M. de, Mansfield, J. W., Grabov, N., Brown, I. R., Ammouneh, H., Tsiamis, G., Forsyth, A., Robatzek, S., Grant, M., and Boch, J. 2006. Pseudomonas syringae effector AvrPtoB suppresses basal defence in Arabidopsis. Plant J. 47:368-382.

Vierstra, R. D. 2009. The ubiquitin-26S proteasome system at the nexus of plant biology. Nat. Rev. Mol. Cell Biol. 10:385-397.

Zhou, B., Mural, R. V., Chen, X., Oates, M. E., Connor, R. A., Martin, G. B., Gough, J., and Zeng, L. 2017. A subset of ubiquitin-conjugating enzymes is essential for plant immunity. Plant Physiol. 173:1371-1390. 